\section{Orbital floor triamcinolone acetonide injections for the management of active non-infectious uveitis}

M Roesel, M Gutfleisch, C Heinz, B Heimes, $B$ Zurek-Imhoff and A Heiligenhaus
Department of

Ophthalmology,

St Franziskus Hospital,

University Duisburg-Essen,

Muenster, Germany

Correspondence:

A Heiligenhaus,

Department of

Ophthalmology,

St Franziskus Hospital,

University Duisburg-Essen,

Hohenzollernring 74,

Muenster 48145, Germany

Tel: + 490251933080

Fax: +4902519330819.

E-mail: arnd.heiligenhaus@

uveitis-zentrum.de

Received: 23 November 2007

Accepted in revised form:

25 March 2008

Published online: 9 May

2008

The authors have no financial interest in any of the reagents used in this study

\begin{abstract}
Purpose To evaluate the effect of orbital floor triamcinolone acetonide (OFTA) injections on inflammation and visual acuity in active non-infectious uveitis.

Methods Monocentre, retrospective study in 94 eyes (86 patients) with acute non-infectious uveitis receiving a single OFTA $(40 \mathrm{mg})$ injection. Outcome measures were anterior chamber (AC) and vitreous cell counts, laser flare photometry, best-corrected visual acuity (BCVA), and complications over a 6-month follow-up period.

Results The number of patients with $\geqslant 1+$ cells in AC and vitreous was significantly lower after treatment than at baseline (each, $P<0.01$ at the follow-up examinations). The anti-inflammatory effect lasted for the 6-month follow-up period. Laser flare photometry values were also significantly lower than the baseline levels at $3(P<0.01)$ and $6(P=0.034)$ months. BCVA did not differ before and after treatment. Macular oedema was reduced in $27 \%$, unchanged in another $59 \%$, and worse in 14\%. Within 6 months, ocular hypertension was observed in up to $8 \%$ and progressing cataract was noted in $29 \%$.

Conclusions OFTA injections improved AC and vitreous inflammation, but the effect on visual acuity and macular oedema was limited. Eye (2009) 23, 910-914; doi:10.1038/eye.2008.114; published online 9 May 2008
\end{abstract}

Keywords: uveitis; triamcinolone; orbital floor; intravitreal; inflammation

\section{Introduction}

Triamcinolone acetonide (TA) is frequently used in uveitic eyes to treat intraocular inflammation or uveitic macular oedema. Intravitreal TA may reduce macular oedema and improve visual acuity, ${ }^{1-6}$ and posterior subtenon TA injections have been suggested for the treatment of inflammation in uveitic eyes. ${ }^{2,3,7-9}$ However, such injections carry a considerable risk for intraocular pressure (IOP) elevation and cataract formation in both of these conditions. ${ }^{1-10}$

In the past, the steroids methylprednisolone and $\beta$-methasone were injected into the orbital floor to treat inflammation, ${ }^{11-13}$ whereas for TA no data are available.

In this study, we analysed our experience with orbital floor triamcinolone acetonide (OFTA) administration for the management of acute ocular inflammation in patients with noninfectious uveitis. The effect of OFTA injection on the inflammatory cells in the anterior chamber (AC) or anterior vitreous, laser flare photometric values, course of visual acuity, and the typical complications were evaluated.

\section{Patients and methods}

In this monocentre study, the charts from 86 patients (94 eyes) treated for active noninfectious anterior, intermediate, or posterior uveitis or panuveitis with OFTA injections were retrospectively reviewed.

Patients with active uni- or bilateral uveitis, even if they were being treated with topical and systemic corticosteroids or systemic second-line immunosuppression, were included. Active uveitis was defined by the presence of cells $(\geqslant 1+$ cells $)$ in the AC or anterior vitreous. In patients with anterior uveitis, TA injections were only given in the presence of cells in the vitreous with or without cystoid macular oedema (CME). 
Before treatment, a comprehensive medical examination was conducted to detect any associated infectious or systemic disease. Patients with infectious uveitis were excluded. None of the patients had shown a tendency for developing steroid-induced intraocular hypertension when treated with prednisolone acetate $1 \%$ eye drops five times daily for at least 2 weeks. The immunosuppressive treatment was not modified in any way nor were any other surgical interventions or TA injections carried out over a period of at least 6 weeks before receiving the study treatment.

Triamcinolone acetonide (Volon A, Bristol-Myers Squibb) was used for all injections. For OFTA administration, $40 \mathrm{mg}$ of TA (in a volume of $1 \mathrm{ml}$ ) were injected through the temporal third of the inferior lid using a 27-gauge needle (20 $\mathrm{mm}$ length).

Standardized evaluations were performed at a baseline examination within 2 weeks prior to treatment and at follow-up examinations 1, 3, and 6 months after injection. These examinations included BCVA evaluation (LogMAR), IOP measurement (Goldmann tonometry), slit-lamp examination, laser flare photometry (Kowa FM-500, Japan), and ophthalmoscopy.

Inflammation in the AC was graded according to the grading pattern described by Hogan et al, ${ }^{14}$ which matches for AC activity with the recently published standards of the SUN working group. ${ }^{15}$ As the degree of inflammation and opacities in the visual axis cannot be distinguished when using standardized photographs and comparing vitreous haze ${ }^{16}$ cells in the lacunae of the anterior third of the vitreous cavity were enumerated. Lens status was determined using slit-lamp examination after pupil dilatation. Nuclear, cortical, and posterior subcapsular cataract was documented on a scale from 0 to 3. Cataract progression was defined as increased or new opacification. The presence of CME was confirmed by fluorescein angiography (FA). Perifoveal leakage of the dye that accumulates in cystic spaces in a petaloid pattern, continuing to diffuse positive staining at the fovea in the late frames, was diagnosed as CME. The FA pictures were evaluated independently by two observers in a masked fashion. Macular leakage noted after about $3 \mathrm{~min}$ was rated semi-quantitatively as reduced, unchanged, or worse as compared to baseline examination.

Data were analysed using MedCalc Version 6.0. The $\chi^{2}$-test and Fisher's exact test for categorical data and Wilcoxon test, Mann-Whitney $U$-test, and Student's $t$-test were used for statistical analysis, when appropriate. $P<0.05$ was judged as statistically significant.

\section{Results}

Epidemiological data are listed in Table 1. The most common systemic diseases were sarcoidosis,
HLA-B27-associated uveitis, chronic inflammatory bowel disease, and multiple sclerosis. Before treatment, $27 \%$ of the patients were on second-line immunosuppressive drugs, which were not modified during the reported follow-up after OFTA injection. The most commonly administered drugs were methotrexate, cyclosporine, and azathioprine. In $30 \%$ of the patients, oral prednisolone was given at a mean dose of $3.7 \mathrm{mg}$ daily.

\section{Influence on inflammation}

The effect of OFTA on AC and vitreous cell count and on laser flare photometry is summarized in Table 2. Mean number of cells in the AC and vitreous decreased after the injection. The number of patients with $\geqslant 1+$ cells in the AC was significantly lower over the complete followup period than at baseline ( $P<0.01$ at 1,3 , and 6 months). The number of patients with $\geqslant 1+$ vitreous cells after treatment was also significantly lower than before injection ( $P<0.01$ at each follow-up examination).

The mean laser flare photometry values decreased after OFTA from 33.2 photons per second at baseline to 25.3 photons per second at the 6-month examination. Flare values at 3 and 6 months were also markedly lower $(P<0.01$ and $P=0.034)$.

\section{Influence on BCVA}

BCVA at baseline and during the course of follow-up is summarized in Table 3. Compared with baseline, mean

Table 1 Epidemiological data of patients treated for noninfectious uveitis with a single orbital floor triamcinolone acetonide injection

\begin{tabular}{lc}
\hline Epidemiologic data & $\begin{array}{c}\text { Orbital floor TA } \\
(\mathrm{n}=94)\end{array}$ \\
\hline Mean age $( \pm \mathrm{SD})$ & $42.6 \pm 17.0$ \\
Male:female $(n)$ & $36: 58$ \\
Mean visual acuity (LogMAR) & $0.45(\mathrm{SD} 0.36)$ \\
Second-line systemic immunosuppressive & $27 \%$ \\
drugs & \\
Systemic corticosteroids & $n=28(30 \%)$ \\
& \\
Anatomic uveitis form & $n=27(29 \%)$ \\
$\quad$ Anterior & $n=27(29 \%)$ \\
Intermediate & $n=4(4 \%)$ \\
Posterior & $n=26(28 \%)$ \\
Panuveitis & $n=18(19 \%)$ \\
& $n=6$ \\
Associated systemic immune-mediated diseases & $n=6$ \\
Sarcoidosis & $n=2$ \\
HLA B 27 & $n=4$ \\
Chronic inflammatory bowel disease & \\
Multiple sclerosis & \\
\hline
\end{tabular}

Abbreviations: HLA, human leukocyte antigen; TA, triamcinolone acetonide. 
Table 2 Treatment for non-infectious uveitis with a single orbital floor triamcinolone acetonide injection

\begin{tabular}{|c|c|c|c|c|}
\hline & Before & After 1 month & After 3 months & After 6 months \\
\hline Anterior chamber cells ${ }^{a}$ & $56 \%$ & $13 \% *$ & $21 \% *$ & $19 \% *$ \\
\hline Mean cell count $( \pm S D)$ & $0.89 \pm 0.85$ & $0.24 \pm 0.50$ & $0.28 \pm 0.50$ & $0.30 \pm 0.56$ \\
\hline Vitreous cells ${ }^{\mathrm{b}}$ & $81 \%$ & $32 \% *$ & $30 \% *$ & $19 \% *$ \\
\hline Mean cell count $( \pm$ SD) & $1.49 \pm 1.01$ & $0.63 \pm 0.89$ & $0.52 \pm 0.72$ & $0.38 \pm 0.71$ \\
\hline Laser flare photometry (photons per second, SD) & $33.2 \pm 26.8$ & $32.5 \pm 27.2$ & $22.3 \pm 14.4^{*}$ & $25.3 \pm 16.6^{*}$ \\
\hline
\end{tabular}

${ }^{*} P<0.05$ as compared to baseline.

${ }^{a} \geqslant 1+$ cells in $1 \times 1 \mathrm{~mm}$ in the anterior chamber as detected by slit lamp.

${ }^{b} \geqslant 1+$ in $1 \times 1 \mathrm{~mm}$ in lacunae of anterior $1 / 3$ of vitreous cavity as detected by slit lamp.

Table 3 Treatment for non-infectious uveitis with a single orbital floor triamcinolone acetonide injection

\begin{tabular}{|c|c|c|c|c|}
\hline & Before & After 1 month & After 3 months & After 6 months \\
\hline Visual acuity ( $\log M A R \pm S D)$ & $0.45 \pm 0.36$ & $0.41 \pm 0.42$ & $0.46 \pm 0.39$ & $0.39 \pm 0.38$ \\
\hline $\mathrm{IOP} \geqslant 22 \mathrm{~mm} \mathrm{Hg}$ & $2 \%$ & $5 \%$ & $3 \%$ & $8 \%$ \\
\hline Cataract progression & - & ND & ND & $29 \%$ \\
\hline Cataract incidence & $38 \%$ & ND & ND & $55 \% *$ \\
\hline
\end{tabular}

Abbreviations: IOP, intraocular pressure; ND, not detected.

${ }^{*} P<0.05$ as compared to baseline.

Best-corrected visual acuity (BCVA, mean $\pm \mathrm{SD}$ ) before and after injection, cataract progression (in aphasic patients), and ocular hypertension (over $21 \mathrm{~mm} \mathrm{Hg}$ ).

BCVA was nearly unchanged after injection. The observations at baseline and after treatment were not significantly different $(P=0.15, P=0.95, P=0.15$ at 1,3 , and 6 months). In $30 \%$ of the treated eyes, we observed an increase in BCVA by about two or more lines. Visual acuity remained unchanged in another $59 \%$ of the treated eyes and deteriorated by about two or more lines in $11 \%$. Patients in whom vision improved by about two or more lines had significantly worse baseline BCVA $(P<0.01)$.

\section{Influence on cystoid macular oedema}

According to the results of fluorescein angiography, CME was reduced in $27 \%$, although it remained unchanged in $59 \%$ and had even worsened in $14 \%$.

\section{Side effects from the injection}

Complications occurring within the 6-month follow-up are listed in Table 3. Although an increase in IOP to above $21 \mathrm{~mm} \mathrm{Hg}$ was observed in up to $8 \%$ of eyes, cataract progression was found in $29 \%$. After 6 months, the cataract incidence was greater than at baseline $(P=0.048)$. No further complications occurred after TA injection in any of the patients in this study. In particular, none of the patients developed endophthalmitis, retinal detachment, ocular perforation, or bleeding.

\section{Discussion}

This study evaluates the effect of injecting TA into the orbital floor to treat acute non-infectious uveitis, an issue that has not been specifically addressed in the literature to date. In the present study, the markedly reduced AC and vitreous cell counts demonstrate a good antiinflammatory effect for this mode of depot steroid delivery.

After transtenon retrobulbar TA infusion, Okada ${ }^{17}$ reported a clinical efficacy of $96 \%$ for vitritis, $82 \%$ for $\mathrm{CME}$, and $33 \%$ for posterior retinal vasculitis.

Ozkiris ${ }^{16}$ did not detect significant differences between intravitreal TA and systemic prednisolone for treating vitreous inflammation with regard to vitreous haze.

By using laser flare photometry AC inflammation can be measured objectively and quantitatively. ${ }^{18}$

Guex-Crosier et $a l^{19}$ found reduced flare after systemic steroid administration. However, no data are currently available from laser flare photometric studies after TA administration in uveitic eyes. We observed significantly decreased flare after injecting TA into the orbital floor. Protein accumulation was reduced in the AC, indicating stabilization of the blood-aqueous barrier after OFTA administration. In our study patients, second-line immunosuppressive drugs could not be reduced after TA injection, as other authors have suggested. ${ }^{4}$

Over the follow-up period, visual acuity only increased in $30 \%$. These results do not reflect the encouraging data reported for intravitreal or posterior 
subtenon administration of TA. ${ }^{1,2,4-7,9,16,17}$ For IVTA, improvement in BCVA was reported in 50-60\%. The better efficacy of IVTA could be attributed to the higher intravitreal TA concentrations that were previously detected after intravitreal drug injection as compared to a posterior periocular injection. ${ }^{20}$ However, in contrast to the aforementioned studies, OFTA was administered for inflammatory activity and not to treat macular oedema in our study. We observed a proportion of patients in whom visual acuity deteriorated $(11 \%)$ in the course of the follow-up. Persisting RPE changes due to chronic macular oedema and cataract progression could be responsible for the lack of BCVA improvement. Interestingly, patients in whom vision improved by about two or more lines had had significantly worse baseline BCVA. Multivariate analysis did not detect further variables (cataract development, CME, or inflammation) that influenced results.

A positive impact on CME was obtained in only $27 \%$, and notably, the CME did not completely disappear in any of the treated patients. Compared to the data for intravitreal TA administration in the literature, which suggest complete resolution of the CME in a higher number of eyes, ${ }^{1,2,5,6}$ these results are disappointing.

It has often been reported that ocular hypertension was a common problem after intravitreal TA injections. ${ }^{2,4-6,10,16}$ Data concerning hypertension after posterior subtenon administration of TA are conflicting. Although $\mathrm{Kuo}^{8}$ reported ocular hypertension in nearly $79 \%$ of patients after subtenon injection, Okada ${ }^{17}$ observed ocular hypertension in $27 \%$ after transtenon retrobulbar infusion. Ferrante ${ }^{12}$ found no differences in ocular hypertension after orbital floor methylprednisolone-acetate and posterior subtenon TA injection. In the present study, we observed an increase in ocular pressure to over $21 \mathrm{~mm} \mathrm{Hg}$ in only $8 \%$. No patient required glaucoma surgery. In all patients with elevated IOP, topical glaucoma medication or systemic acetazolamide could successfully control IOP.

Cataract progression is another common complication of intravitreal TA treatment. Previous studies reported that lens opacification progressed in $20-29 \%, 1,5$ and Okada $^{17}$ observed cataract progression in $31 \%$ after transtenon retrobulbar TA injection. In our study, cataracts progressed in as many as $29 \%$ over the 6-month follow-up period. There were no further side effects from TA injection, however; particularly, no ocular penetration, endophthalmitis, retinal detachment, or ocular trauma was observed.

In conclusion, OFTA is effective in managing inflammatory activity in the AC and vitreous. The impact on visual acuity and macular oedema was poor. However, fewer side effects were reported after OFTA than after intravitreal TA administration.
Randomized controlled clinical trials are required to further evaluate the clinical effectiveness of the different TA delivery routes in uveitic eyes.

\section{References}

1 Androudi S, Letko E, Meniconi M, Papadaki T, Ahmed M, Foster CS. Safety and efficacy of intravitreal triamcinolone acetonide for uveitic macular edema. Ocul Immunol Inflamm 2005; 13: 205-212.

2 Angunawela RI, Heatley CJ, Williamson TH, Spalton DJ, Graham EM, Antcliffe RJ et al. Intravitreal triamcinalone acetonide for refractory uveitic cystoid macular oedema: longterm management and outcome. Acta Ophthalmol Scand 2005; 83: 595-599.

3 Jea SY, Byon IS, Oum BS. Triamcinolone-induced intraocular pressure elevation: intravitreal injection for macular edema and posterior subtenon injection for uveitis. Korean J Ophthalmol 2006; 20: 99-103.

4 Kok H, Lau C, Maycock N, McCluskey P, Lightman S. Outcome of intravitreal triamcinolone in uveitis. Ophthalmology 2005; 112: 1916.

5 van Kooij B, Rothova A, de Vries P. The pros and cons of intravitreal triamcinolone injections for uveitis and inflammatory cystoid macular edema. Ocul Immunol Inflamm 2006; 14: 73-85.

6 Young S, Larkin G, Branley M, Lightman S. Safety and efficacy of intravitreal triamcinolone for cystoid macular oedema in uveitis. Clin Experiment Ophthalmol 2001; 29: 2-6.

7 Helm CJ, Holland GN. The effects of posterior subtenon injection of triamcinolone acetonide in patients with intermediate uveitis. Am J Ophthalmol 1995; 120: 55-64.

8 Kuo HK, Lai IC, Fang PC, Teng MC. Ocular complications after a sub-tenon injection of triamcinolone acetonide for uveitis. Chang Gung Med J 2005; 28: 85-89.

9 Yoshikawa K, Kotake S, Ichiishi A, Sasamoto Y, Kosaka S, Matsuda H. Posterior sub-Tenon injections of repository corticosteroids in uveitis patients with cystoid macular edema. Jpn J Ophthalmol 1995; 39: 71-76.

10 Jonas JB, Sauder G, Budde WM, Spandau UH, Kamppeter BA. Triamcinolone acetonide-induced ocular hypertension. J Ocul Pharmacol Ther 2006; 22: 247-250.

11 Duguid IG, Ford RL, Horgan SE, Towler HM, Lightman S. Combined orbital floor betamethasone and depot methylprednisolone in uveitis. Ocul Immunol Inflamm 2005; 13: 19-24.

12 Ferrante P, Ramsey A, Bunce C, Lightman S. Clinical trial to compare efficacy and side-effects of injection of posterior sub-Tenon triamcinolone versus orbital floor methylprednisolone in the management of posterior uveitis. Clin Experiment Ophthalmol 2004; 32: 563-568.

13 Riordan-Eva P, Lightman S. Orbital floor steroid injections in the treatment of uveitis. Eye 1994; 8(Part 1): 66-69.

14 Hogan MJ, Kimura SJ, Thygeson P. Signs and symptoms of uveitis. I. Anterior uveitis. Am J Ophthalmol 1959; 47: 155-170.

15 Jabs DA, Nussenblatt RB, Rosenbaum JT. Standardization of uveitis nomenclature for reporting clinical data. Results of the First International Workshop. Am J Ophthalmol 2005; 140: 509-516.

16 Ozkiris A. Intravitreal triamcinolone acetonide injection for the treatment of posterior uveitis. Ocul Immunol Inflamm 2006; 14: 233-238. 
17 Okada AA, Wakabayashi T, Morimura Y, Kawahara S, Kojima E, Asano Y et al. Trans-Tenon's retrobulbar triamcinolone infusion for the treatment of uveitis. Br J Ophthalmol 2003; 87: 968-971.

18 Guex-Crosier Y, Pittet N, Herbort CP. Evaluation of laser flare-cell photometry in the appraisal and management of intraocular inflammation in uveitis. Ophthalmology 1994; 101: 728-735.
19 Guex-Crosier Y, Pittet N, Herbort CP. Sensitivity of laser flare photometry to monitor inflammation in uveitis of the posterior segment. Ophthalmology 1995; 102: 613-621.

20 Inoue M, Takeda K, Morita K, Yamada M, Tanigawara Y, Oguchi Y. Vitreous concentrations of triamcinolone acetonide in human eyes after intravitreal or subtenon injection. Am J Ophthalmol 2004; 138: 1046-1048. 